\title{
Can Jellyfish Dream? Conceptual Representations in Unsupervised Generative Learning
}

\author{
Serge Dolgikh * \\ National Aviation University, Kyiv (Ukraine)
}

\begin{abstract}
Representations play essential role in learning of artificial and biologic systems by identifying characteristic patterns in the observed environment. In this work we examine unsupervised latent representations of image data with a collective of generative neural network models. A convolutional autoencoder with strong redundancy reduction was used to create low-dimensional latent representations of a dataset of geometrical shapes. The structure of the resulting latent representations was studied comprehensively with several methods, including density clustering, direct visualization, generative probing and scanning. It was demonstrated that conceptual representations with good decoupling of principal concepts can be produced with generative models of limited complexity; that latent representations have high level of consistency between individual learners; and that the resulting latent representations have a well-defined geometrical and topological structure correlated with principal patterns in the observable data. The results of this work support the hypothesis that conceptual latent representations can emerge naturally in unsupervised generative learning under certain essential constraints and can be a natural platform for emergence of some intelligent functions and behaviors.
\end{abstract}

\section{KEYWORDS}

Artificial Intelligence,

Concept Learning,

Clustering,

Representation

Learning,

Unsupervised Learning

\section{INTRODUCTION}

EPRESENTATION learning with the objective to identify the informative patterns in the observable data has a well-established record in the field of Machine Learning. Identification of stable patterns of attribute combinations, or concepts [1], in the observed environment can provide significant advantages to the learner due to significant reduction in the cost of processing of sensory inputs, memory, as well as the ability to associate beneficial behaviors to a general class of observations, rather than specific observed instance.

For these reasons, intelligent systems capable of successful identification of concepts in the observable data find applications in increasing number of domains. In the commonly used approach, concepts can be identified in the process of representation learning [2] that produces a transformed representation of observable data in a latent space created by a learning model in the process of training under certain constraints.

\section{A. Related Work}

Hierarchical representations of observable data were obtained in a completely unsupervised training process with Restricted Boltzmann Machines (RBM) and Deep Belief Networks (DBN) [3],[4] offering a noticeable improvement in the quality of subsequent supervised leaning. Different types, architectures and flavors of generative models were investigated since including autoencoder neural networks, Generative Adversarial Networks (GAN) and other ([5]-[9] to name only a few in a rapidly expanding field), resulting in improved accuracy and versatility of the models with virtually unlimited range of applications. In the theory of learning systems, the relations between learning and statistical thermodynamics was studied in [10],[11] leading to understanding of a deep connection between learning processes in artificial neural models and principles of information theory and statistical thermodynamics.

In experimental studies, a number of interesting results was reported, including: the "cat experiment", demonstrating spontaneous emergence of concept sensitivity on a single neuron level in unsupervised deep learning with a massive dataset of images [12]. Disentangled representations were produced and discussed in [13] with deep variational autoencoder architecture and different types of visual data, pointing at the possibility of a general nature of the effect. Concept-associated structure in artificial neural networks was observed in the representations of generative models with different types of real-world data as Internet traffic [14], anomaly detection [15], medical and aerial surveillance imaging [16],[17], linguistics [18] and a range of generative architectures [19],[20], pointing to a possibility of a deep connection between unsupervised generative learning and characteristic structures in the latent representations of learning models that can be interpreted as general concepts in the observable data.

Over the decades, a growing number of approaches and directions in concept learning were developed, that would require a separate work to cover in sufficient detail: version-space learning [21], based on selection of candidates in the concept classes by matching attributes to the rules or "hypotheses" of concept classes; a wide range of representation-based approaches based on different types and architectures of artificial neural network models discussed earlier, that demonstrated possibility to learn a broad range of concepts in data of different types confidently and with smaller training samples than in conventional supervised learning. Some methods specifically aimed to build up on strategies characteristic in human learning, such as learning the letters in the alphabet (such as Omniglot challenge [22] and others).

A major challenge for the attribute-driven methods can be noisy real-world data and complex data types described by very large 
number of observable parameters common in realistic applications, resulting in rules of increasing complexity; whereas representationbased methods, successful in a number of specific cases could be challenging to extend to different types of observable data and may require considerable domain knowledge, such as training data with explicitly defined concepts.

In reinforcement learning [23], concepts can be interpreted as general classes of the intelligent agent states and actions produced via application of the rules and constraints of the reward-based training. Approaches and methods were developed to obtain generalized concepts of states and actions in reinforcement learning, such as tile-coding, temporal abstraction [24] and many others. It can be noted that training process in reinforcement learning is driven by the external knowledge of the domain being learned in the form of the reward feedback, and for this reason concepts identified with these methods must bear some relation to this knowledge.

In more recent years, concept learning entered the domain of practical applications in the rapidly growing field of robotics. The already stated advantages of potentially vast reduction of memory and processing power while improving the effect of the response via association of actions and behaviors to a general class of observable inputs drove development of methods and approaches in practical applications of concept learning [25] however this area is too broad and diverse to attempt to discuss here.

Interestingly, while as mentioned earlier, a range of results in generative learning of artificial systems demonstrated that structure emergent in the latent representations created by generative models in the process of unsupervised self-learning can be used as a foundation for learning methods and processes based on distillation of characteristic patterns in the observable environment, they were paralleled very recently by advances in investigation of biologic sensory networks [26],[27] that demonstrated commonality of lowdimensional neural representations in processing sensory information by mammals, including humans. These results suggest intriguing parallels in learning processes of artificial and biologic systems.

To summarize this brief overview of a wide and rapidly expanding field of general concept learning, while significant progress has been made in interpretation and learning of concepts in different environments, with growing number of models, methods and applications with increasing efficiency, there remain some essential limitations in our understanding how general, abstract concepts emerge and are learned. The first relates to the generality of results obtained with models of increasing specialization and / or complexity, where it can be questioned whether the observed effects related to specific selection of model architecture or properties, and to what extent they can be expected to be applicable to different models and data in a more general way.

The second question, of conceptual "bootstrap" relates to the fact that to observe, measure and learn higher-level concepts models in these studies needed confidently identified instances of the concepts. This knowledge was not a part of the learning model and had to be provided externally. Then the question of origin of concepts becomes less clear; whether observations of concept-related structure of information in the learning models has general character; or is it related to a specific choice of concepts selected for experiments?

* Corresponding authors:

E-mail address: mail@mail.com
Accordingly, an investigation of the origins of general concepts would benefit from development of methods and approaches that do not rely in any essential way on the external knowledge about the observable distribution, including in the form of concept-labeled data, to investigate and identify characteristic structure in the representations of observable data produced by learning systems.

In this work we attempted to approach both of these questions first by using generative models of limited complexity, well within the range of simplest biologic systems and without advanced or specific architectural features. The models that were used are based on a standard convolutional autoencoder with strong dimensionality reduction in the layer producing the latent representation. The size of the studied models roughly corresponded to a neural system of a jellyfish [28],[29]. By using common neural network models of limited complexity, the problem of generality of the results can be addressed in some way, and it can be expected that the observed effects could be reproduced with models and data of similar complexity.

To address the "bootstrap" problem, methods of analysis of latent representations were developed that are entirely unsupervised, do not depend and do not require any data or otherwise information of external concepts. These unsupervised methods can be used to study and describe the "native" information structure in the latent representations of generative models and thus allow to advance toward a solution to the bootstrap problem, that is, a description of the emergent structure in the latent representations that does not depend on external definition of concepts.

The structure of this document is as follows: section II provides a description of the models, architecture, data and the methods used in this work; in section III the results on the structure and topology of unsupervised latent representations obtained with models of unsupervised generative learning are reported. Section IV provides a summary of the results along with a discussion, including the origins of abstraction in generative learning and connections to fields and areas in machine and general learning. Section $\mathrm{V}$ provides a synthesis of the work and the conclusion.

\section{MethodS}

A convolutional autoencoder [30] with strong dimensionality reduction to a compact three-dimensional latent representation with the data of greyscale images of geometric shapes was used to produce and study structured low-dimensional latent representations and conceptual models of a simple visual environment as described in this section.

\section{A. Convolutional Autoencoder Model}

A convolutional autoencoder model had the encoding stage with convolution-pooling layers followed by several layers of dimensionality reduction with the encoding layer of size 3 (i.e., a three-dimensional latent representation defined by activations of neurons in the encoding layer).

The dimensionality of the latent layer was chosen based on the recent results pointing at low dimensionality of representations of visual sensory data in the human sensory cortex [26] as well as for convenience of evaluation and observation of the resulting latent representations. The decoding, or generative stage was fully symmetrical to the encoder. Overall, the architecture of models used in the experiments had 21 layers and approximately 40,000 trainable parameters.

The diagram and description of the generative model is given in Figure 1 and Table 1 . The models were implemented in Keras / 
Tensorflow [31] with a number of common machine learning and data processing packages and libraries used in the analysis and visualization of latent representations.

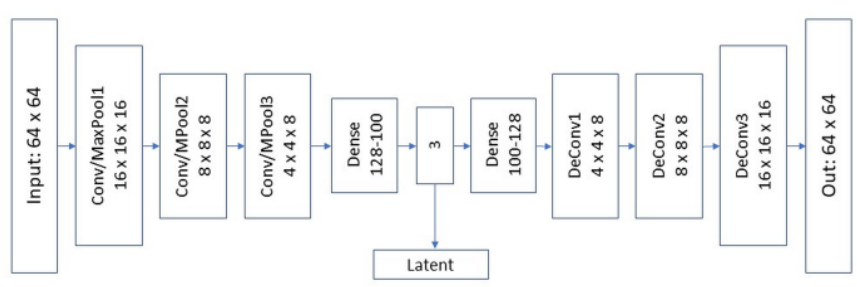

Fig. 1. Convolutional autoencoder with dimensionality reduction.

TABLE I. MODEL PARAMETERS

\begin{tabular}{ccccc}
\hline Parameter & $\begin{array}{c}\text { Convolution } \\
\text { stages }\end{array}$ & $\begin{array}{c}\text { Deep } \\
\text { layers }\end{array}$ & $\begin{array}{c}\text { Total } \\
\text { parameters }\end{array}$ & $\begin{array}{c}\text { Cost } \\
\text { function }\end{array}$ \\
\hline Value & $2-3$ & 5 & c. $10^{4}$ & $\begin{array}{c}\text { binary cross- } \\
\text { entropy }\end{array}$ \\
\hline
\end{tabular}

The models were trained in an unsupervised process without labeled data for minimization of average distance between training samples and the output generated by the model and thus represent a subclass of unsupervised generative learning systems.

\section{B. Data}

To investigate the capacity of models to learn concepts in the process of unsupervised generative training, three datasets of images of simple geometric shapes with increasing variety and complexity were used. Given the previously discussed analogy with primitive biologic systems, the shapes used in the experiments can be interpreted as: positive inputs e.g. food; negative inputs, like predators; and general background.

The first, smaller dataset denoted "Ideal-Small" (Id-S) consisted of greyscale images of circles, triangles and greyscale backgrounds of size $(64 \times 64)$, with two representative samples per class with difference in the size and contrast of fore / back-ground, for the total of 300 images.

The second dataset, "Ideal-Medium" (Id-M) contained the total of $600-1,000$ greyscale images of circles, triangles and backgrounds with variation in size in the range $0.3-1.0$ of the image size (that is, $0.3 \times 64$ pixels), with variation of contrast of fore- vs. background for each size.

In the two "ideal" datasets the images were centered, symmetrical and had no rotation. Only darker foregrounds relative to the background were used.

The third dataset, "Real-Large" (Real-L) contained 1,500 images of randomly generated colored geometric shapes and backgrounds varying in: size; position relative to the center of the image; rotation; and the color of the fore- and background. The images were obtained from 2D geometric shapes dataset [32] and processed as follows:

1. Convert to greyscale.

2. Select: triangles and circles of size greater than approximately 0.3 of the image size $(0.3 \times 64$ pixels $)$, with a darker foreground.

3. Select: shapes of size less than approximately 0.15 of the image size.

4. Other images were filtered out.

The images in p. 2 represented the instances of the classes of interest in realistic observations, whereas those in p.3 represented realistic backgrounds.

Additionally, to imitate real-world observations, tests with randomly generated images varying in size and contrast were used.

\section{Training}

The models were trained in an unsupervised process to minimize the difference between the training batches of images in the input and their regenerations produced by learning models in the output layer (generative error). In unsupervised autoencoder training [3] this difference, measured by the cost functional is backpropagated through the layers of the network to achieve minimization of generative error with standard methods of training, such as Stochastic Gradient Descent [33].

A success of generative learning following the unsupervised training of models with a dataset of images as described earlier was verified by the change in the value of the cost function (i.e., mean generative error) during training and the ability of the learner to generate a random subset of images of the types represented in the training dataset (Figure 2). Most models were successful in generative learning, though a spread in the generative quality was observed, not unlike in a group of learning individuals.

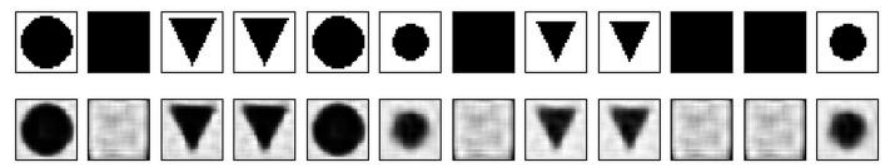

Fig. 2. Convolutional autoencoder with dimensionality reduction.

Following the objective of the study to investigate the structure of latent representations as well as the capacity to generalization, individual models were trained in two groups:

- Models trained with the Ideal-Small dataset: starting / final validation loss, after $25-50$ training epochs: $0.69 /<0.5$.

- Models trained with the Ideal-Medium dataset: starting / final validation loss, after $25-50$ training epochs: $0.69 / \sim 0.55$.

After the completion of the training phase, models of both types showed good generative capacity with random samples from the training dataset (Fig. 2).

\section{RESULTS}

The experiments were conducted with generative models in the groups Ideal-S and Ideal-M trained with unsupervised datasets as described in the previous sections. Training was conducted in entirely unsupervised mode and no labeled samples were used in configuration or training of the models. This approach ensures that the observed effects are not related to a specific choice of higherlevel concepts or any other form of external information about the data.

For the experiments, models in the better segment of training metrics were selected, based on the value of the final validation cost in unsupervised learning and generative ability.

\section{A. Structural Consistency of Latent Representations}

The models in both groups showed remarkable consistency between learning individuals in the structure of created latent representations. Given that training process contained an essential element of randomness, such as selection of samples in training batches, these results indicate that the structure that emerged in the latent representations as a result of unsupervised generative learning reflected some essential patterns and characteristics in the observable data.

The plots in Figure 3 show visualizations of a test subset of samples in the latent representations of two individual, independently trained models. A clear similarity of structure can be observed between latent representations of individual learners. 

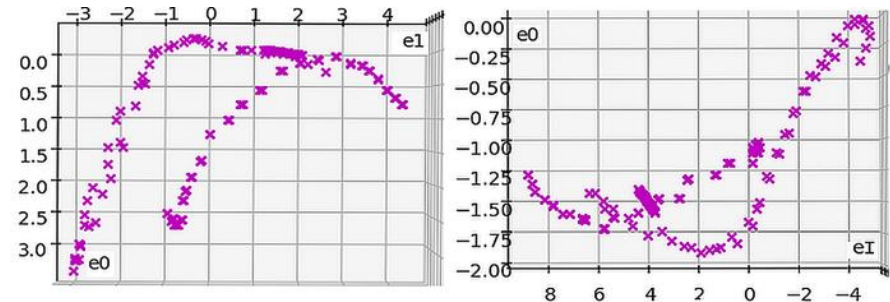

Fig. 3. Latent representations (models: Ideal-M)

The results of visual analysis were confirmed by application of a density clustering method (MeanShift, [34]) in the latent representations of the models. It produced arrays of density clusters with similar characteristics in models trained with the same dataset (Table II).

TABLE II. DENSITY STRUCtURE IN LATENT REPRESENTATIONS

\begin{tabular}{lcccc}
\hline Model & $\begin{array}{c}\text { Visual } \\
\text { similarity }\end{array}$ & $\begin{array}{c}\text { Number, } \\
\text { clusters }\end{array}$ & $\begin{array}{c}\text { Population, } \\
\text { clusters }{ }^{\text {a }}\end{array}$ & $\begin{array}{c}\text { Correlation, } \\
\text { cluster / shape }\end{array}$ \\
\hline Id-S, $\mathbf{1}$ & yes & 4 & $0.2-0.38$ & yes \\
Id-S, 2 & yes & 4 & $0.2-0.38$ & yes \\
Id-S, $\mathbf{3}$ & yes & 5 & $0.16-0.38$ & yes \\
Id-M, 3 & yes & 7 & up to 0.33 & yes \\
Id-M, 2 & yes & 6 & up to 0.33 & yes \\
\hline
\end{tabular}

${ }^{a}$ As fraction of a general sample

The clusters in the latent representations of trained models showed clear correlation with the types of images (i.e., shapes) that models were exposed to in training (Figure A1, Appendix).

The consistency of latent structure observed between different individual learning models indicates that the structure of the representation is defined by the characteristic patterns in the observable data and possibly, architecture of the model and is relatively stable between individual instances of learning models.

\section{B. Generalization and Abstraction}

In this group of experiments, models trained with one dataset were presented with data from different datasets to evaluate their capacity to generalize data that was not observed in training.

The models in the first group, Ideal-S were trained with data composed of two instances per class, differing in size and contrast. Test samples given to these models in the experiments had significantly wider variation by each of the characteristics: size and contrast (sets Ideal-M, Real-L); position relative to the center of the image and orientation (set Real-L), allowing to evaluate how well the models were able to infer those characteristics in generative selflearning. The success or otherwise, of interpretation of these characteristics was evaluated visually from the quality of generation of randomly selected samples from the test data, as illustrated in Figure 4.

As was observed in the experiments, the models were generally successful in generalizing the characteristics of size and contrast even when trained with only a handful of representative samples per type of shape (Figure 4, top rows). Though the training set Ideal-S had only two combination of size / contrast per type of shape, trained models were generally successful in interpreting shapes of different sizes and contrasts in the test subset of Ideal-M.

On the other hand, these less sophisticated models were mostly unable to interpret correctly shifted or rotated shapes.

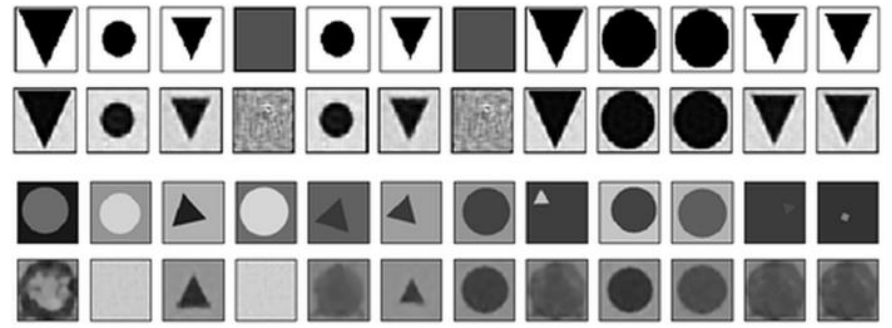

Fig. 4. Abstraction of size, contrast, position and orientation (top rows: model Id-S, test set Id-M; bottom rows: model Id-M, test set Real-L).

Models trained with a more representative set, Ideal-M, were generally successful in interpreting such shapes (Figure 4, bottom rows) even though they were not present in the set the models were exposed to in training. Note that samples with negative contrast, i.e. lighter foreground relative to background were not present in the training sets and were not used in the evaluation, though it can be noted that in a number of examples the models were able to interpret such samples successfully as well. The results of these experiments are summarized in Table III.

TABLE III. Generalization of Size; Contrast; Position and Rotation

\begin{tabular}{ccccc}
\hline Model & $\begin{array}{c}\text { Size (set: } \\
\text { Id-M) }\end{array}$ & $\begin{array}{c}\text { Contrast } \\
\text { (Id-M) }\end{array}$ & $\begin{array}{c}\text { Position } \\
\text { (set: } \text { Real-L) }\end{array}$ & $\begin{array}{c}\text { Rotation } \\
\text { (Real-L) }\end{array}$ \\
\hline Id-S & yes & yes & no & no \\
Id-M & yes & yes & often & mostly \\
\hline
\end{tabular}

The results in this section allow to conclude that generative models of limited complexity are well capable of generalization of important characteristics of the observable data such as: size; contrast; position; and orientation even when trained with minimal variation of samples.

\section{Topology of Latent Representations}

Visualizations of the distribution of images in the latent representation created by models in the previous section pointed at well-defined character of latent regions associated with characteristic types of images in the observable data as connected, continuous areas with clear boundaries. This observation is confirmed by more detailed analysis of the latent concept regions.

To investigate the structure of latent representations in an entirely unsupervised mode, a method of latent probing was developed, whereby a trained model received an input as a set of coordinates in the latent region of interest and generated an image associated with the latent position defined by the input coordinates in the observable space by propagating them through the generative component of the model.

Latent probing allowed to study generative structure in the latent representations with virtually unlimited level of detail by moving the latent position of the input in the latent regions of interest and observing the output generated by the model. Several essential findings were made in these experiments.

1. Latent regions associated with characteristic types of data were connected and continuous. A close neighborhood of a latent position producing an image in a certain observable class of shape was likely to produce the observable instance of the same class (Appendix, Fig. A.2); a line between latent points in the same class generated observable images of that class (however it needs to be noted that multiple regions of the same class appear to be possible as discussed further below, so the points must belong to the same topologic region). A central (mean) position of several points in the region of a given shape class is likely to be in the class as well. 
2. Clear boundaries were observed between adjacent regions of different classes of shapes, that produced mixed images with elements of both classes (Fig. 5).
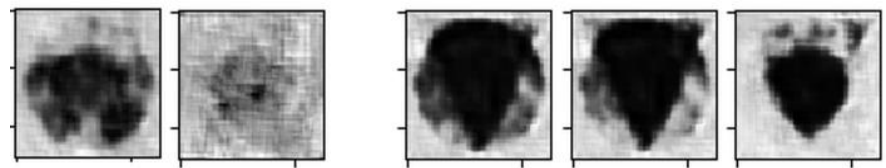

Fig. 5. Boundary areas (left: circles / background regions; right: circles / triangles regions).

Observation of boundary areas between regions of characteristic types of inputs supports the hypothesis of continuous and connected manifold topology in the latent representations.

3. Representative instances, or "prototypes" [35] can be associated with characteristic latent regions via a number of possible strategies. In this work, centers of density clusters calculated with a general, not labeled sample were used. Density-derived latent prototypes identified by this strategy produced generated images of the shapes associated with the latent region even though they did not represent actually observed images (Figure A.4, Appendix). The clusters appeared to be associated with characteristic attributes of shapes, such as size and contrast.

These results suggested that as a result of unsupervised generative training the models produced latent representations in the form of well-defined regions associated with characteristic types of inputs in the training dataset. This structure was produced in a completely unsupervised process guided only by the imperative to minimize generative error under the constraints imposed in training and can be interpreted as "native" or natural concepts in the observable data, that emerge naturally to satisfy the constraints of generative learning.

The emergent native concept structure, or framework, then allows to associate an observed sample $X$ to a native concept class $T_{i}$ via the relation of containment of the encoded position of $X$ to the latent region $K_{i}$ of the native concept:

$$
r_{X}=E(X) \in K_{i} \rightarrow X \in T_{i}
$$

where $E: O \subset R$, the encoding transformation from the observable space $O$ to the latent representation $R$ performed by a trained generative model (Fig.1). In this work the relation of containment was defined by applying clustering to an encoded general sample in the latent space.

It is worth noting that generative models were able not only to classify an observation as one of the learned native concepts (1), but also, importantly, to interpret symbolic concept tokens as representative instances of the concept. This ability clearly differentiates generative models from conventional methods of supervised classification where such a task would not be meaningful. The mapping of a symbol $T_{i}$ associated with a concept $P$ to an observable instance of the concept can be achieved straightforwardly by propagating the latent prototype of $T_{i}$ through the generative submodel:

$$
P T_{i}=G t_{i}=G\left(t T_{i}\right)
$$

where $G: R \subset O$, the generating transformation from the latent representation to the observable space of inputs performed by the generative model; $t_{i}$, a representative instance, or latent prototype of the concept $T_{i}$. The strategy of association of latent prototypes, $t(T)$ to concept regions can be static, for example, as a dictionary of pairs (Region, Prototype instance(s)), or dynamic, where representative instances in the concept regions are calculated on each invocation. In this work, centers of clusters identified by a density clustering method were used as representative instances of concepts, that is a type of a static association unless density clusters are recalculated.

\section{Generative Structure of Latent Representations}

As discussed in the preceding section, unsupervised generative learning that creates structured latent representations associated with characteristic patterns in the observable data allows to decouple encoding and generative stages of the model in processing stimuli from the environment.

In observation, the function of the encoder is to transform the observed input to a latent position, defined by the latent structure created in training with the incentive to minimize the error of generation of the observable sample from its encoded position in the latent representation. The generating model, on the other hand, transforms a position in the latent space back to the observable space, creating an "interpretation" of observation by the learning model. Importantly, as follows from (2), after the training phase completes and the encoding and generative parts of the models have been created, the interpretation does not need to be associated with an actual observation, such as an encoded input, but can be triggered by any activation of a latent position, however it was caused.

To verify this hypothesis, the method of latent probing was extended to perform "scanning" of the latent space of pre-trained models, providing a clear and complete description of the emergent conceptual structure in latent space and generative capacity of the model. The method was defined and implemented as follows:

1. A rectangular grid of the latent dimensionality created with the dimensions of the encoded general sample in the latent representation; the step between the nodes and the dimension of the grid being the parameters of the method.

2. The nodes of the latent grid traversed sequentially with latent probing applied at each node.

3. For each node, the latent position is propagated through the generative sub-model (2), creating a generated image of the latent position of the node in the observable space.

4. The resulting hypercube of images generated by the model at the nodes of the latent grid fully described generative capacity of the model in the latent region of the grid.

Examples of "generative scans" obtained for the models trained with different datasets are presented in Figure 6. Characteristic regions associated with different types of shapes are clearly seen in the scans for models of all studied types, having the appearance of broad slices or layers with distinct boundary areas.

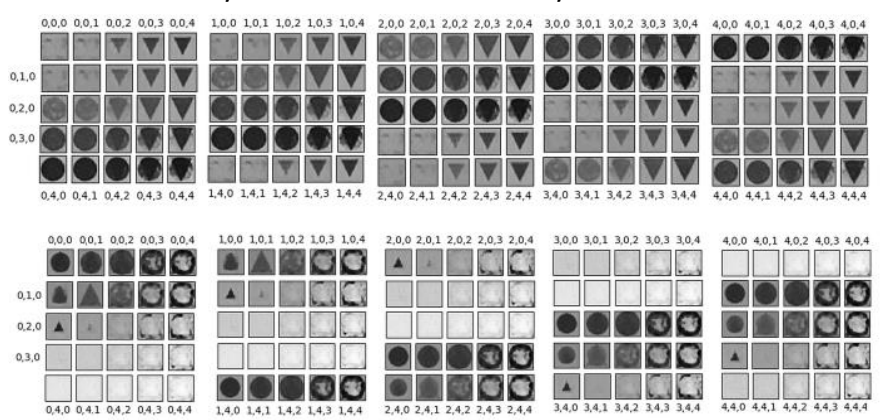

Fig. 6. Generative scans of latent representations (top: model Id-S, bottom: model Id-M; 3D latent grid with 5 regularly-positioned nodes per axis).

The methods of latent probing and latent scanning can be applied in an entirely unsupervised process and do not require any data prelabeled with known concepts. For this reason, they are suitable for investigation of the conceptual structure emergent in unsupervised 
representations of generative models without dependence on external definition or descriptions of the concept and thus allow to advance in resolving the "bootstrap" problem in investigation of origins of general concepts.

The results of application of methods of latent probing and latent scanning with individual models of different types supported the conclusions of the previous sections on consistency of latent representations and on connected and continuous topology of concept regions in the latent space.

An interesting effect was observed in the latent region denoted " $R$ " of Ideal-M models trained with more representative dataset of shapes (Fig.7, bottom plot, areas $(0,2,3-2,0,4)$ and $(1,4,3-4,1,4)$ ). Generated observable image in this latent region had a clear appearance of a ring, a shape that was not present in the training dataset and was not seen by the model in training. This effect appears to be stable and was observed with several different model instances trained independently with the same dataset (Fig. 7) that allows to conclude that generation of this specific type of a shape must have some intrinsic relationship to the observable data and the architecture of the models used in the experiments.

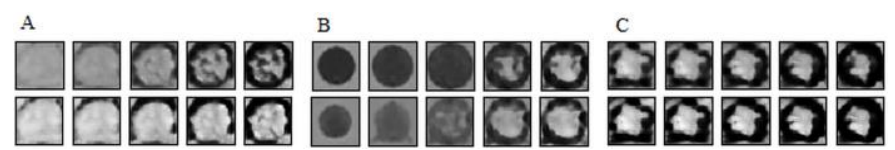

Fig. 7. Latent region " $R$ ", models Id-M.

\section{DISCUSSION}

The analysis of latent representations obtained with models of unsupervised generative learning in this work resulted in a number of essential observations. The experiments confirmed that structured conceptual representations can be produced by unsupervised generative models of limited complexity with plain architecture providing arguments for a general nature of the effect. The complexity of studied generative models was well within the range of primitive biologic organisms, comparable to that of jellyfish, snails and leeches, in the range of $10,000-30,000$ neurons [29], providing independent evidence for the hypothesis that intelligent behaviors can be more common in biologic systems [36]. An interesting result of the methods developed in the study was the ability to observe native concept prototypes or "natural categories" [35] directly in the latent representations of investigated models, as representative instances of latent regions associated with characteristic types of sensory inputs.

By applying unsupervised methods in the analysis of latent representations it was demonstrated that generative learning can leads to emergence of well-defined latent regions associated with essentially different types of observable data, providing empirical support for the manifold assumption [37], commonly used in semisupervised and unsupervised learning. These results points to possible origin of higher-level abstract concepts in unsupervised generative learning under the constraints of generative accuracy and redundancy reduction, both having clear evolutionary advantage for the learners, regardless of the origin, artificial or biologic.

The results discussed in Section III.B may shed the light on the origins of abstraction of different characteristics of observable data, such as: size, contrast, position and rotation. It was demonstrated that even less sophisticated models, exposed to minimal variation of data have some capacity to abstract certain characteristics of observable data, for example, size and contrast. As was commented, generative learning creates a mapping between the latent representation and the observable space, defined by the generative part of the model. An interpretation of an input presented to a trained model is made by the encoder that determines the latent position to which an observed input will be placed. That decision is guided by the expectation of which encoded position of the input would result in smaller generative error between the input and the generated image of its encoded position produced by the generative model.

With simpler models trained with minimal data (Ideal-S), the models were able to abstract the sizes of shapes that they were not shown in training by guessing that encoding a shape to the region associated with a similar type (like a smaller triangle to the standard triangle in the training dataset) would produce smaller generative error than placing it in the region associated with a different, "wrong" shape (circle or background). However, these models were often confused by rotated and shifted shapes because confident estimation of generative error from a small variety of samples in the training set was difficult or not possible as different choices could produce similar error (for example a rotated mid-size triangle to a larger triangle vs. a similar size circle). Extending the training dataset with greater variety of samples in size and contrast allowed more precise estimation of generative error and models trained with a more representative dataset Ideal-M were mostly successful in generalizing not only size and contrast, but also position and rotation (to a certain extent) of the shapes.

Thus, abstract concepts of shape can emerge naturally as a result of constraints of generative accuracy and redundancy reduction in unsupervised generative learning.

These results also suggest an observation on evolution of biologic systems. As is evident from the results of Section III.A, neural network models even of limited complexity can be highly successful in identification of basic images especially with a clear, direct and centered position of observation. Then, it could be more practical for a learning system to develop more sophisticated mechanisms of focusing and positioning to obtain higher quality sensory inputs, than significantly increase the size and complexity of the processing neural network for more confident identification. Indeed, many of primitive biologic systems have well-developed mechanisms of orientation and focus, likely developed under the incentive to improve recognition of sensory inputs.

It was demonstrated in the experiments in Sections III.A, III.D that latent representations of models trained with similar data were consistent between individual models. This observation points to a possibility that latent conceptual structure emergent in generative self-learning could provide a natural basis for sharing semantic information about the environment in a collective of learners, via exchange of symbols associated with latent representations of common patterns in the observed environment [38]. The ability of generative models to produce conceptual prototypes, or representative instances of native concepts discussed in Section III.C can provide a natural basis for such strategy of collective learning. Further investigation may deliver new interesting results in this area of research.

The results of generative scanning (Section III.D) can offer essential insights into the semantics of latent coordinates. It can be observed that the latent axes indeed can have a meaning essential to encoding general characteristics of commonly observed patterns, such as size, contrast and type of the shape. However as can be concluded from detailed analysis of generative scans produced by models in the study, the semantics did not appear to have a global nature in the latent space but were determined by the latent position. The same latent axis at different positions could control the size of a shape or 
the contrast, or transition to a different concept region. These results again point to the conclusion of a manifold-like topology of latent regions in which latent coordinates have locality, that is, region and position-dependent semantics. From this perspective, further detailed studies of topology in latent representations can deliver interesting and promising results.

It was commented earlier that conceptual latent representations allow not only generalization of inputs by characteristics essential to the learner but also decoupling of the input from its interpretation. Indeed, as was demonstrated with generative scans, any activation in the latent space can produce an observable image, or perception, just like an actual observation. The decoupling allows the learner to create an internal, conceptual framework of the observed environment. In most cases generated perceptions represented types of inputs that are familiar to the system from unsupervised generative training. However, as was observed in Section III.D, stable regions can form in the latent representations producing generative interpretations that had no direct analogies in the observable data. Could one infer from these experiments that artificial generative models of the size and complexity comparable to the intelligence of a jellyfish can indeed produce perceptions of forms that did not exist in their sensory environments?

A limitation of this study that was in part intended, was limited complexity of the architecture of the models comparable to neural complexes of simple biologic organisms. It allowed to evaluate structure and topology of the resulting latent representations, however, the number and complexity of characteristic patterns in the observed data was kept to the minimum. It is possible that successful learning of more complex and variable visual and other sensory data would require more sophisticated neural network architectures and produce more complex latent structures. It is the intent of the future studies to investigate more complex and advanced architectures including size; depth; optimization features and other factors in unsupervised concept learning in more complex sensory environments.

\section{CONCLUSION}

The capacity to learn general concepts and associate with them intelligent behaviors can offer a clear and strong evolutionary advantage in massive reduction of required memory and complexity of processing of sensory inputs and for that reason is likely to be an important selection factor in an evolutionary process. The results of this work demonstrated that conceptual representations can be produced by neural networks of standard "vanilla" architecture of limited complexity as a natural result of unsupervised observation of the environment under the constraints that are both natural and common for learning systems, whether of artificial or biologic origin.

The methods of unsupervised analysis of latent representations produced in unsupervised generative learning under such constraints developed in this work allowed to confirm that conceptual representations with latent regions associated to characteristic patterns in the observable data can emerge naturally and predictably in this process. The findings obtained with methods of unsupervised analysis of latent representations, including latent probing and scanning allowed to advance in understanding and resolution of the conceptual bootstrap problem in the area of conceptual representations and conclude that latent structure that emerges in the process of unsupervised generative learning can be a natural foundation and framework for simple abstract concepts, defined by the constraints of accuracy and compression (i.e., redundancy reduction) in generative self-learning.
Methods of generative analysis of latent representations developed in the study allowed to advance in understanding of origin and drivers of abstraction and generalization ability and functions in generative learning, as a general consequence of principles of information processing in such systems. It can be an essential factor and a basis for a number of intelligent functions and behaviors, including the ability to associate symbolic information about observations to characteristic latent structures, such as conceptual prototypes discussed in this work, and share it in a collective of learners with similar architecture of processing sensory inputs.

For these reasons, in our view, investigation of conceptual representations obtained in unsupervised generative learning merits further attention of the research community.

\section{APPENDIX}

\section{A.1. Latent Distributions of Shape Classes}

The diagrams show distribution of latent positions of a test subset of images in identified shape classes by different independently trained generative models.
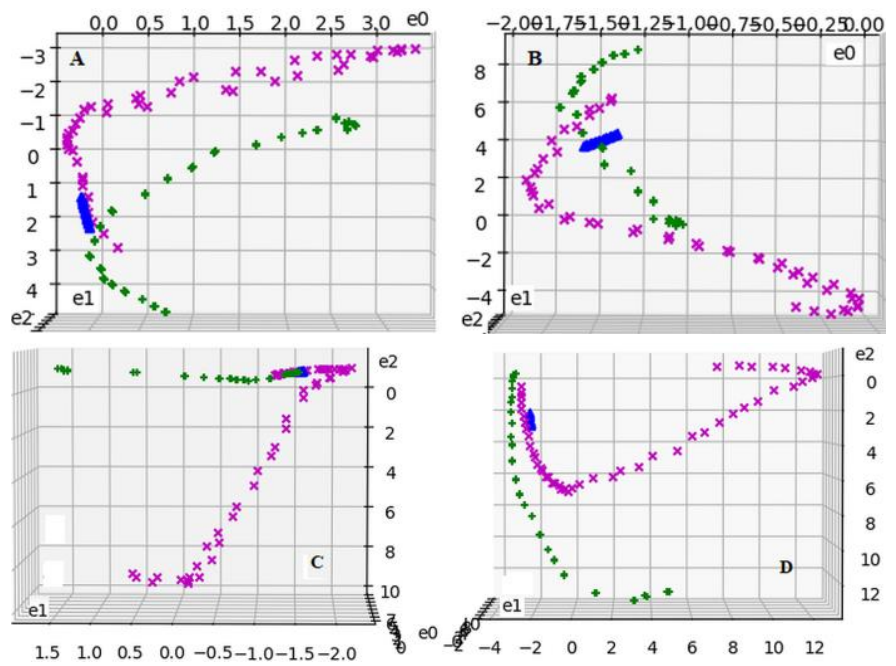

Fig. A.1. Latent distributions of shape classes, models Id-M (A, B); Id$S(C, D)$. Legend: magenta: circles; green: triangles; blue: background.

\section{A.2. Latent Probing and Topology of Latent Space}

To verify connectedness and continuity of latent regions images were generated from a close neighborhood of a selected latent position (position of origin) in a concept region with latent probing (Figure A.2.).

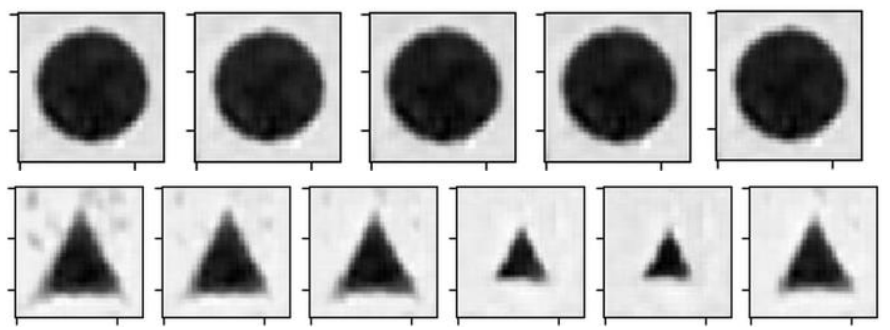

Fig. A.2. Connectedness of latent class regions. Top row: position of origin (leftmost) in the region " $\mathrm{C}$ " (circles); bottom row: region " $\mathrm{T}$ " (triangles).

\section{A.3. Semantics of Latent Coordinates}

Semantics of latent axes were investigated with latent probing by producing generated images from positions of origin in concept regions along latent axes. 


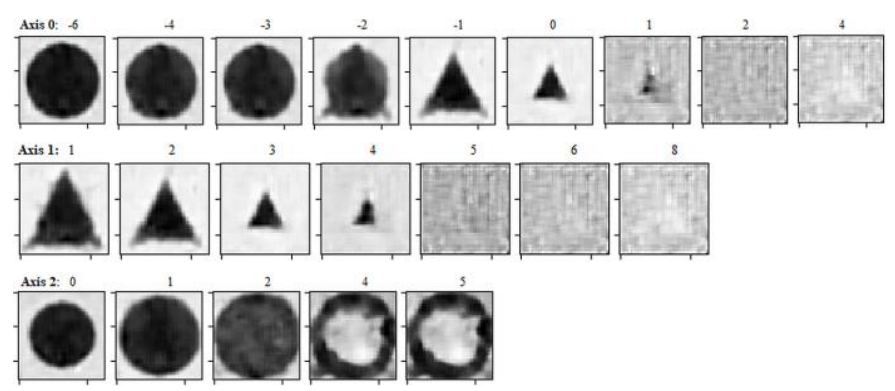

Fig. A.3. Generative semantics of latent coordinates. Top to bottom rows: variation of generated output along latent axes $0-2$.

\section{A.4. Conceptual Prototypes}

Latent prototypes were obtained as centers of density clusters identified with Mean Shift density clustering. Observable prototypes were obtained by generating images of latent prototypes with the generative submodel of the autoencoder. While density clustering is a relatively crude method of selection of representatives in concept regions, it illustrates that relatively simple approaches allow to identify characteristic instances for characteristic concept regions with entirely unsupervised methods.

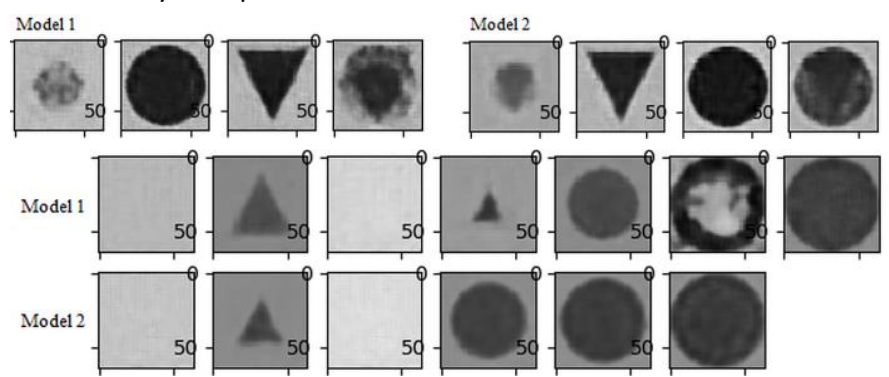

Fig. A.4. Observable concept prototypes. Top: model Id-S; bottom: IdM.

\section{REFERENCES}

[1] J.S. Bruner, J.J. Goodnow, J.J. and G. A. Austin, A study of thinking, New York, USA: John Wiley and Sons, 1956.

[2] Y. Bengio, A. Courville, P. Vincent, P., "Representation Learning: a review and new perspectives", arXiv:1206.5538 [cs.LG], 2014.

[3] G.E. Hinton, S. Osindero, Y.W. Teh, "A fast learning algorithm for deep belief nets", Neural Computation, vol. 18, no. 7, pp. 1527-1554, 2006.

[4] A. Fischer, C. Igel, "Training restricted Boltzmann machines: an introduction", Pattern Recognition vol. 47, pp. 25-39, 2014.

[5] Y. Bengio, "Learning deep architectures for Al", Foundations and Trends in Machine Learning, vol. 2, no. 1, pp. 1-127, 2009.

[6] A. Coates, H. Lee, A.Y. Ng, "An analysis of single-layer networks in unsupervised feature learning", in: 14th International Conference on Artificial Intelligence and Statistics (AISTATS), Lauderdale, FL, USA, 2011, vol. 15, pp. 215-223.

[7] M. Welling, and D.P. Kingma, "An introduction to variational autoencoders, Foundations and Trends in Machine Learning, vol. 12, no. 4, pp. 307-392, 2019.

[8] A. Creswell, T. White, V. Dumoulin, K. Arulkumaran, B. Sengupta and A.A. Bharath, "Generative adversarial networks: an overview", IEEE Signal Processing Magazine, vol. 35, no. 1, pp. 53-65, 2018.

[9] Partaourides, H., Chatzis, S.P., "Asymmetric deep generative models", Neurocomputing, vol. 241, pp. 90-96, 2017.

[10] G. E. Hinton and R.S. Zemel, "Autoencoders, minimum description length and Helmholtz free energy", Advances in neural information processing systems, vol. 6 pp. 3-10, 1994.

[11] Ranzato, M.A., Y.-L. Boureau, S. Chopra and Y. LeCun, "A unified energybased framework for unsupervised learning", in: 11th International Conference on Artificial Intelligence and Statistics (AISTATS), San Huan,
Puerto Rico, 2007, vol. 2, pp. 371-379.

[12] Q.V. Le, M.A. Ransato, R. Monga, M. Devin, K. Chen, G. S. Corrado et al., "Building high level features using large scale unsupervised learning", arXiv 1112.6209 [cs.LG], 2012.

[13] I. Higgins, L. Matthey, X. Glorot, A. Pal, B. Uria, C. Blundell et al., "Early visual concept learning with unsupervised deep learning", arXiv 1606.05579 [cs.LG], 2016.

[14] S. Dolgikh, "Spontaneous concept learning with deep autoencoder", International Journal of Computational Intelligence Systems, vol. 12, no. 1, pp. 1-12, 2018.

[15] C. Zhou and R.C. Paffenroth, "Anomaly detection with robust deep autoencoders", in: 23rd ACM SIGKDD International Conference on Knowledge Discovery and Data Mining, Halifax, Canada, 2017, pp. 665674.

[16] L. Gondara, "Medical image denoising using convolutional denoising autoencoders", in: 16th IEEE International Conference on Data Mining Workshops (ICDMW), Barcelona, Spain, 2016, pp. 241-246.

[17] P. Prystavka, O. Cholyshkina, S. Dolgikh, D. Karpenko, "Automated object recognition system based on aerial photography", in: 10th International Conference on Advanced Computer Information Technologies (ACIT), Deggendorf, Germany, 2020, pp. 830-833.

[18] S. C. A P, S. Lauly, H. Larochelle, M. M. Khapra, B. Ravindran et al., "An autoencoder approach to learning bilingual word representations", in: 27th International Conference on Neural Information Processing Systems (NIPS'14), Montreal, Canada, 2014, vol. 2, pp. 1853-1861.

[19] R.C. Rodriguez, S. Alaniz and Z. Akata, "Modeling conceptual understanding in image reference games", in: Advances in Neural Information Processing Systems, Vancouver, Canada, 2019, pp. 1315513165.

[20] Q. Wang, S. Young, A. Harwood and C.S. Ong, "Discriminative concept learning network: reveal high-level differential concepts from shallow architecture", in: 2015 International Joint Conference on Neural Networks (IJCNN), Killarney, Ireland, 2015, pp. 1-9.

[21] T.M. Mitchell, "Generalization as search", Artificial Intelligence, vol. 18, pp. 203-226, 1982.

[22] B. M. Lake, R. Salaz and J. B. Tenenbaum, "The omniglot challenge: a 3year progress report", Current Opinion in Behavioral Science, vol. 29, pp. 97-104, 2019.

[23] J. Shi, J. Xu, Y. Yao and B. Xu, "Concept learning through deep reinforcement learning with memory augmented neural networks", Neural Networks, vol. 110, pp. 47-54, 2019.

[24] R.S. Sutton, D. Precup and S. Singh, "Between MDPs and semi-MDPs: a framework for temporal abstraction in reinforcement learning", Artificial Intelligence, vol. 112, pp. 181-211, 1999.

[25] J. Nevens, P. Van Eecke and Beuls K., "From continuous observations to symbolic concepts: a discrimination-Based Strategy for Grounded Concept Learning", Frontiers in Robotics and Al, vol. 7, no. 84, 2020.

[26] T. Yoshida and K. Ohki, "Natural images are reliably represented by sparse and variable populations of neurons in visual cortex", Nature Communications, vol. 11, p. 872, 2020

[27] X. Bao, E. Gjorgiea, L.K. Shanahan, J. D. Howard, T. Kahnt, J. A. Gottfried, "Grid-like neural representations support olfactory navigation of a twodimensional odor space", Neuron, vol. 102 no. 5, pp. 1066-1075, 2019.

[28] G. Roth and U. Dicke, "Evolution of the brain and intelligence", Trends in Cognitive Science, vol. 5, p. 250, 2005.

[29] A. Garm, Y. Poussart, L. Parkefelt, P. Ekström and D-E. Nilsson, "The ring nerve of the box jellyfish Tripedalia cystophora", Cell and Tissue Research, vol. 329, no. 1, pp. 147-157, 2007.

[30] Q.V. Le, "A tutorial on deep learning: autoencoders, convolutional neural networks and recurrent neural networks", Stanford University, 2015.

[31] Keras: Python deep learning library. Accessed: Jan. 21, 2021. [Online]. Available: https://keras.io/.

[32] A. El Korchi, "2D geometric shapes dataset", Mendeley Data, V1, 2020, doi: $10.17632 /$ wzr2yv7r53.1.

[33] J. C. Spall, Introduction to stochastic search and optimization: estimation, simulation, and control. Hoboken, New Jersey: Wiley, 2003 (ISBN 0-47133052-3).

[34] K. Fukunaga and L. D. Hostetler, "The estimation of the gradient of a density function, with applications in pattern recognition", IEEE 
Transactions on Information Theory, vol. 21 no. 1, pp. 32-40, 1975.

[35] E. H. Rosch, "Natural categories", Cognitive Psychology, vol. 4, pp. $328-$ 350, 1973.

[36] T. E. Feinberg and J. Mallatt, "The nature of primary consciousness. A new synthesis", Consciousness and Cognition, vol. 43, pp. 113-127, 2016.

[37] Zhou X., Belkin M.: Semi-supervised learning. In: Academic Press Library in Signal Processing Elsevier, vol. 1, pp. 1239-1269, 2014.

[38] S. Dolgikh, "Synchronized conceptual representations in generative learning", Cambridge Open Engage, 2020, doi:10.33774/coe-2020-rshht. 